To appear in the International Journal of Remote Sensing

Vol. 00, No. 00, Month 20XX, 1-17

\title{
Retrieval of Phenological Stages of Onion Fields During the First Year of Growth by means of C-band Polarimetric SAR Measurements
}

\author{
L.Mascolo $^{a}$, J.M. Lopez-Sanchez ${ }^{b}$ F. Vicente-Guijalba ${ }^{b}$, G.Mazzarella $^{a}$, F. Nunziata ${ }^{c}$ \\ and M.Migliaccio ${ }^{c *}$ \\ ${ }^{a}$ Dipartimento di Ingegneria Elettrica ed Elettronica, Università di Cagliari \\ Piazza D'Armi, 09123 Cagliari, Italy; \\ ${ }^{b}$ Department of Physics, System Engineering and Signal Theory, University of Alicante \\ Campus of Sant Vicent del Raspeig, 03690 Sant Vicent del Raspeig, Alacant, Spain; \\ ${ }^{c}$ Dipartimento di Ingegneria Università degli Studi di Napoli Parthenope \\ Centro Direzionale Isola C4, 80143, Napoli, Italy.
}

(v4.1 released May 2013)

\begin{abstract}
The phenological stages of onion fields in the first year of growth are estimated using polarimetric observables and single-polarization intensity channels.

Experiments are undertaken on a time series of RADARSAT-2 C-band fullpolarimetric SAR images collected in 2009 over the Barrax region, Spain, where ground truth information about onion growth stages is provided by the ESA-funded AgriSAR field campaign conducted in that area.

Experimental results demonstrate that polarimetric entropy or copolar coherence when used jointly with the cross-polarized intensity allows unambiguously distinguishing three phenological intervals.
\end{abstract}

Keywords: Agriculture; Onion; Phenology; SAR polarimetry; Phenology estimation.

\section{Introduction}

Onion (Allium Cepa L.) is recognized as one of the most important vegetable crops worldwide (Opara 2003).

In this study, the potential of C-band polarimetric Synthetic Aperture Radar (SAR) measurements in estimating phenological stages of onion fields is first investigated. In order to provide an exhaustive understanding of growth stages of onion, it is important to introduce some basic terminology about phenology.

Crop phenology denotes the continuos development of agricultural crops during the cultivation cycle, i.e. from sowing or transplanting to harvest, and it is usually expressed by means of numerical scales (Meier 2001; Zadoks et al. 1974). Phenological stages of crops are generally grouped into three main phases: vegetative, reproduction and maturation. The vegetative phase comprises those stages which represent the initial development of plants, starting with the growth from seed

*Corresponding author. Email: migliaccio@uniparthenope.it 
and finishing with fully developed plants. The reproductive phase includes inflorescence emergence and flowering stages, while the maturation phase goes from the development of fruits to plants ripening and senescence.

Onion is a biennial crop (Lee et al. 2013), i.e. a crop whose lifecycle has a twoyears duration. In the first year of growth, a sown onion seed evolves to a plant and then the bulb starts to grow. Once grown, the bulb overwinters and then, during the spring and the summer of the next year (second year of growth), the onion plant flowers and produces seeds (Lee et al. 2013).

Therefore, the phenological stages of onion fields during the first year of growth are all comprised in the vegetative phase of the plant development, while the reproduction and the maturation phases are observed in the second year of growth.

When onion growing is devoted to bulb production for food purposes, plants are usually harvested at the end of the vegetative phase, thus being transformed in annual crops. In this case, the knowledge of phenological stages represents a key information that can be effectively used for the planning and management of those cultivation practices aimed at improving the food crop production. On the other hand, when dealing with seeds production (for onion as well as other vegetable alliums) it is really important to understand how vegetative growth and bulbing interact with the flowering stages, for a successful production of seeds (Brewster 2008). As a consequence, the monitoring of onion growth stages in the first growing season turns out to be important also in this case. Hence, the interest of this paper arises from the importance of the vegetative phase of onion for both food and seeds production, and its final objectives are the monitoring and the estimation of such phenological stages by means of meaningful observables provided by C-band RADARSAT-2 polarimetric SAR (polSAR) imagery.

Within the framework of active microwave remote sensing some studies, e.g. (Le Toan et al. 1989; Mattia et al. 2003), have demonstrated the relationship between the $\mathrm{X}$ - and $\mathrm{C}$ - band radar backscattering at different polarization channels and the growth stages of crops.

The monitoring of crop phenology by means of SAR remote sensing has gained more interest with the launch of space-borne SAR sensors capable of measuring polarimetric scattering in a coherent way, such as RADARSAT-2 (C-band), TerraSAR-X (X-band) and the most recently launched Sentinel-1 (C-band). Although the issue of the long revisit time (24 days for RADARSAT-2) has to be faced by combining different beams and ascending/descending orbits, recent studies (Lopez-Sanchez et al. 2011, 2012, 2013, 2014; Liu et al. 2013; Mascolo et al. 2014; Vicente-Gujialba et al. 2014a,b; De Dernanrdis et al. 2014) have shown the potential of polSAR measurements to estimate growth stages of agricultural fields in a robust and efficient way.

In literature, phenology estimation by polSAR data has been explicitly addressed in (Lopez-Sanchez et al. 2012, 2013, 2014; Vicente-Gujialba et al. 2014a,b; De Dernanrdis et al. 2014) where effective retrieval procedures based on supervised classification (Lopez-Sanchez et al. 2012, 2013, 2014) and dynamical systems concept (Vicente-Gujialba et al. 2014a,b; De Dernanrdis et al. 2014) have been proposed. In (Lopez-Sanchez et al. 2012, 2014) five phenological intervals of rice fields were effectively estimated by means of proper sets of X-band dual-pol and C-band quadpol parameters derived from single acquisitions. At X-band (Lopez-Sanchez et al. 2012) the eigenvector/eigenvalues decomposition parameters (Cloude and Pottier 1996, 1997) were used along with the coherence and the phase difference between the copolar channels while, only the eigenvector/eigenvalues decomposition parameters were used at C-band (Lopez-Sanchez et al. 2014). 
In this study, the same methodology used in these two works, based on a detailed analysis of different polarimetric observables, is employed to estimate the phenological stages of onion fields during the first year of growth.

A dense time series of Single Look Complex (SLC) fine quad-pol RADARSAT2 images collected over the Barrax region, Spain, from April to September 2009 is used. Gound truth information about onion growth stages is provided by the ESA-funded Agricultural bio/geophysical retrieval from frequent repeat pass SAR and optical imaging (AgriSAR) field campaign conducted in Barrax almost concurrently with the SAR acquisitions. It is important to underline that the same SAR data were previously used in (Moran et al. 2012), where the sensitivity of the backscattering coefficients to growth stages of onion, barley, wheat, and alfalfa fields was analyzed. The present study is intended to complement that work because here the interpretation of the radar response of onion fields is based on exploiting the whole polarimetric space (i.e. correlations, decompositions outputs) and not only on the backscattering coefficients.

\section{Phenological Stages of Onion in the First Year of Growth}

In this section we describe the phenological stages of onion plants during the first year of growth, i.e. the vegetative phase of the plant development. Such a phase comprises different growth stages, from the germination to the maturation of the bulb or bulb ripening.

During germination the plant and the radicles evolve from the seed and, at the end of this stage, the cotyledon (seed leaf) emerges through the soil surface with a looplike shape (Brewster 2008). When germination is completed, the leaf development stage occurs during which the plant grows its leaves (up to seven). Once the seventh leaf is appeared, the first falls and the plant goes into the start of bulbing stage (Brewster 2008). During this stage, while the second and the third leaves desiccate, the bulb begins to extend and more leaves (from the eighth to the thirteenth leaf) appear and the plant reaches its maximum height.

The next stage is the bulb swelling stage, characterized by a rapid growth of the bulb and the desiccation of the fourth-sixth leaf. Moreover, the leaves may bend or fold. This stage is then followed by the fall down stage, in which the weight of the foliage leads it to collapse. Finally, in the bulb ripening stage, the outer skin of the bulb becomes dry and the foliage desiccates.

\section{Test site, Ground Measurements and SAR Data}

Barrax belongs to the province of Albaceteprovince of Albacete (Spain) and is located on the La Mancha plateau at $700 \mathrm{~m}$ above the sea level. Due to the presence of many agricultural fields, the Barrax area has been used as a test site where several remote sensing experiments have been carried out (Berger et al. 2001; GonzálesSanpedro et al. 2005, 2008; Atzbeger and Richter 2012; Moran et al. 2012; Sobrino et al. 2013; Latorre-Carmona et al. 2014).

In the AgriSAR field campaign conducted in 2009 in Barrax more than 100 parcels, i.e. wheat, barley, oat, corn, sweet corn, onion, sunflower, pea and papaver fields, were monitored between the spring and the autumn seasons. Such an intensive field campaign led to two sets of ground measurement. The first one provided information about the phenological stages of all the monitored parcels. The second set of ground measurements started at the beginning of July and was carried out 
only in 23 parcels. It provided additional information about phenology and other kind of information such as plant density, row orientation and crop height. Moreover, meteorological measurements, including precipitation events, wind speed and air temperature, were carried out at meteorological stations present in the Barrax area, and information regarding the irrigation schedule of some fields was also provided.

In this study, we focus on seven onion parcels located in Barrax and its surroundings. These parcels are clearly visible in the Google Earth picture shown in figure 1. Note that parcels E, F and G were also analyzed in (Moran et al. 2012).

Information regarding the phenological stages of the parcels is provided by the main set of ground measurements as reported in table 1, where nine stages are identified. It can be noted that a parcel is in a certain stage in the time window defined by the start and end dates. The start date of a given stage corresponds to the day after the end date of the previous stage. Although some of these dates may be the same for different parcels, in general they differ from one parcel to another. It must be pointed out again that the succession of phenological stages listed in table 1 corresponds to the vegetative phase of the onion parcels since, according to the two years lifecycle that characterizes onions, the presence of both foliage and the bulb growth stages witnesses that the reproductive and the maturation phases do not occur. Therefore, we are observing onion fields in their first year of growth. The seedling stage refers to the growth of the cotyledon (seed leaf) before and after its emergence through the soil, while stages from $S T A G E_{2}$ (2 leaves stage) to $S T A G E_{6}$ (6-7 leaves stage) denote the leaf development stage. Then, the start of bulb growth, the bulb growth and the ripening stages should correspond to the start of bulbing, the bulb swelling and the bulb ripening stages, respectively. Regarding to the last stage, it is reasonable to assume that it also includes the fall down stage. An important aspect that may provide a rough information about the end of the ripening stage is the knowledge of the harvest date. The start date of seedling and the end date of ripening of all the parcels, along with further information recorded by the second set of ground measurements (phenology, harvest date and the row orientation) are listed in table 2, where each date is also expressed in Day of Year (DoY). Note that the start of seedling and the end of ripening are simultaneous for some parcels. The sowing date is not reported since it is not provided for any parcel. Regarding the harvest date, it is provided only for parcels $\mathrm{E}$ and $\mathrm{F}$ (only an approximate date is indicated). If we focus on parcel $\mathrm{A}$ we note that while the end date of the ripening stage is on August 19, further information indicates that the parcel is still in the ripening stage on September 10. For those parcels whose the harvest date is not provided, there is no information, not even approximate, about the the end of the ripening stage.

A further limitation of ground truth information regards the measurements of biophysical parameters. In fact, the only parameters provided by the second set of ground measurements are: plant density (plants $\mathrm{m}^{-2}$ ), height, and shadowing area (referred as plants diameter). Plant density and plants height are provided for all the parcels but parcel C. Regarding plants height, it was recorded only in five days for parcel A (in the bulb-growth and ripening stages) and in four days for the remaining parcels (in the bulb-growth and ripening stages). For each parcel the recorded height value is around $0.5 \mathrm{~m}$. Finally, regarding the shadowing area, it was recorded in the same days in which plants height was recorded and it is always equal to $0.2 \mathrm{~m}$. Unfortunately, this limited amount of data (only 4 or 5 days) does not allow carrying out a meaningful analysis of the polarimetric response of onion fields as a function of biophysical parameters. 
The SAR data set consists of RADARSAT-2 images collected in the Barrax area during the field campaign is used in this study. A time series is built up by combining seven beams, characterized by different incidence angles and different orbit passes (ascending/descending), in order to provide a dense time sampling throughout the growing season. The characteristics of the RADARASAT-2 images are listed in table 3. Unfortunately, four parcels (A, B, C and D) fall outside the coverage of some beams. Hence, for these parcels a smaller subset of images is used. In table 4 the list of the beams that cover a given parcel is reported for all the parcels, along with the number of images analyzed. Note that those images acquired after the end of ripening for parcels B, C, D and G, after September 10 for parcel $\mathrm{A}$, and until two days before the harvest date for parcels $\mathrm{E}$ and $\mathrm{F}$, are not considered. Moreover, images that include man-made objects within a parcel are not considered.

A key aspect of this study is the analysis of the evolution of radar response of the parcels as a function of their phenological stages. In this study a simplified numerical scale is defined to describe phenology observations reported in table 1. Such an ad hoc scale is built by according to the scheme reported in table 5 . In this scheme each start/end date is assigned to a number between 0 and 10 . For instance, let us to consider an onion plant in the seedling stage (STAGE $)$. According to the scheme, the start date of seedling is assigned to " 0 ", while the end date is assigned to "1". Therefore, as the plant evolves continuously in time from the start to the end of this stage, at a given day it will be in a particular state corresponding to a number between 0 and 1 . Regarding the second stage (2 leaves), while the start date of this stage is still assigned to "1" (since it can be assumed that the plant does not evolve very rapidly from one day to the next), the end date is assigned to "2". Hence, while the plant is in the second stage, its state in a certain day is associated with a number between 1 and 2, and so on until the ripening stage ( $S T A G E_{9}$ ). In this specific case, we take full benefit of all the information provided in table 2. For all the parcels, but parcel $\mathrm{A}$, the end date of ripening is considered and assigned to "9". In the case of parcel A the date September 10 is assigned to "9" (see table 2). Moreover, for those parcels whose harvest date is provided (parcels $\mathrm{E}$ and $\mathrm{F}$ ) we define a tenth stage, the pre-harvest stage, by assigning such a date to " 10 ". The resulting phenological scale derived by such a scheme is presented in table 6 . Then, since the radar acquisitions are, in general, not coincident with the ground measurements, a linear interpolation is undertaken in order to derive phenology, in terms of the numerical scale adopted, at the radar acquisition dates (Lopez-Sanchez et al. 2012, 2014).

\section{Analysis of the Polarimetric Observables}

In this section the evolution of the polarimetric observables derived from the RADARSAT-2 SAR data is analyzed, for all the parcels, as a function of phenology and a physical interpretation is provided.

Each image of the time series provides the measurement, for each pixel, of the $2 \times 2$ complex scattering matrix $\mathbf{S}$

$$
\mathbf{S}=\left[\begin{array}{ll}
S_{\mathrm{hh}} & S_{\mathrm{hv}} \\
S_{\mathrm{hv}} & S_{\mathrm{vv}}
\end{array}\right]
$$

where $S_{\mathrm{xy}}$ is the complex scattering amplitude and $\{x, y\}=\{h, v\}$ means that the vertical (v)/horizontal (h) basis is adopted. $\mathbf{S}$ relates the field scattered off the 
scene to the incident one. Note that in (1) reciprocity is assumed.

According to the target vector formalism (Cloude and Pottier 1996), the polarimetric covariance matrix $\mathbf{C}$ is defined as

$$
\mathbf{C}=\left\langle\boldsymbol{k}_{\mathrm{L}} \cdot \boldsymbol{k}_{\mathrm{L}}^{\dagger}\right\rangle=\left[\begin{array}{ccc}
\left\langle\left|S_{\mathrm{hh}}\right|^{2}\right\rangle & \sqrt{2}\left\langle S_{\mathrm{hh}} S_{\mathrm{hv}}^{*}\right\rangle & \left\langle S_{\mathrm{hh}} S_{\mathrm{vv}}^{*}\right\rangle \\
\sqrt{2}\left\langle S_{\mathrm{hv}} S_{\mathrm{hh}}^{*}\right\rangle & 2\left\langle\left|S_{\mathrm{hv}}\right|^{2}\right\rangle & \sqrt{2}\left\langle S_{\mathrm{hv}} S_{\mathrm{vv}}^{*}\right\rangle \\
\left\langle S_{\mathrm{vv}} S_{\mathrm{hh}}^{*}\right\rangle & \sqrt{2}\left\langle S_{\mathrm{vv}} S_{\mathrm{hv}}^{*}\right\rangle & \left\langle\left|S_{\mathrm{vv}}\right|^{2}\right\rangle
\end{array}\right]
$$

where $\boldsymbol{k}_{\mathrm{L}}=\left[\begin{array}{lll}S_{\mathrm{hh}} & \sqrt{2} S_{\mathrm{hv}} & S_{\mathrm{vv}}\end{array}\right]^{T}$ is the target vector in the lexicographic basis (Cloude and Pottier 1996) and ${ }^{\dagger},\langle\cdot\rangle$ and ${ }^{*}$ denote the conjugate transpose, the spatial average and the complex conjugate, respectively.

In this study, $\mathbf{C}$ is estimated, for every pixel, with a spatial average that consists of a $9 \times 9$ sliding boxcar filter. Such a window's size allows obtaining reliable estimates of the polarimetric SAR observables that are used (Lopez-Martinez et al. 2005). Then, each matrix is geocoded (UTM coordinates). Once all the covariance matrices are geocoded, from each of them, the polarimetric coherency matrix $\mathbf{T}$ is obtained, since these two matrices, $\mathbf{T}$ and $\mathbf{C}$, are related by a unitary similarity transformation (Cloude and Pottier 1996). Therefore, for each image of the time series, all the polarimetric observables analyzed in this study are extracted from the covariance and coherency matrices.

Since we are focused on the analysis of onion parcels, regions of interest (ROIs) corresponding to the parcel polygons shown in figure 1 are defined, and the polarimetric observables are estimated within these ROIs. However, an inspection of all the images of the time series, revealed that, in some date, for all the parcels but parcels $\mathrm{C}$ and $\mathrm{G}$ some artifacts were present at the borders of the polygons and also in some zones inside the polygons. Hence, in order to avoid these artifacts, for such parcels a smaller ROI inside the polygons is defined.

\subsection{Backscattering Coefficients and Copolar Ratio}

The evolution of the HH, VV and HV backscattering coefficients, which consist of the diagonal elements of $\mathbf{C}(2)$, is shown as a function of phenology in figure 2(a)$(c)$, respectively. The mean value and the standard deviation evaluated within each parcel is plotted and, at the bottom of the figure, a legend denoting the orbit pass ("A" stands for Ascending and "D" stands for Descending) and the average incidence angle of each beam, along with the mark corresponding to each parcel, is annotated. According to the legend, hereinafter we will refer to a particular beam by the corresponding pass and incidence angle. Note that the same format is adopted in all the subsequent experiments.

We first focus on the backscattering at the copolar channels and their ratio, shown in in figure 2 . We note that, from stages 0 to 2 (seedling-2 leaves stages) their evolution is quite similar, while in terms of absolute values the VV backscattered power is, for most of the parcels, larger than or equal to the $\mathrm{HH}$ one. As a consequence, the copolar ratio, is between -2 and $0 \mathrm{~dB}$. This implies that the scattering from slightly rough/rougher surfaces dominates.

From the 2 leaves to the start of bulb growth (stages 2-7), the HH backscattering coefficient increases, on average, from $\sim-13$ to $\sim-8 \mathrm{~dB}$, while the backscattering at the VV channel increases from $\sim-12$ to $\sim-10 \mathrm{~dB}$. This is mostly due to leaves emergence. In fact, onion leaves emerge in two ranks at $180^{\circ}$ from each other 
(Brewster 2008). As a consequence, the structure of the plants becomes more and more random as new leaves appear and, hence, the scattering from the canopy layer increases. However, in the case of the VV channel such increasing is less significant and the backscattering level is reduced below the $\mathrm{HH}$ one, thus leading to an increase of the copolar ratio. This can be attributed to those leaves whose orientation is almost-vertical (younger leaves) that determine an attenuation of the $\mathrm{VV}$ backscattering coefficient with respect the $\mathrm{HH}$ one.

From the bulb growth to the ripening stage (7-9), the HH backscattering coefficient remains, on average, fairly flat, while the $\mathrm{VV}$ one increases of about $1.5 \mathrm{~dB}$. Hence, the copolar ratio decreases up to values around $1 \mathrm{~dB}$. This can be explained by the more random structure of the plants at these stages, which gives rise to a significant multiple scattering in the canopy layer and, hence, to a large depolarization. In this case, the the VV backscattering coefficient gets closer to the $\mathrm{HH}$ one, resulting in a decrease of the copolar ratio which, theoretically, should be close to 0 dB (Skriver et al. 1999).

Finally, during the pre-harvest stage (9-10) HH (VV) backscattering coefficient increases of about $1.5(2) \mathrm{dB}$. It must be explicitly pointed out that during the acquisition of the beams A23 and A39, rain events took place. Information relevant to weather conditions gathered at the Barrax meteorological station recorded almost $13.7 \mathrm{~mm}$ of rainfall eight hours before the acquisition of the A23 image, and a maximum rainfall of $\sim 1 \mathrm{~mm}$ in the day in which the A39 image was collected.

Concerning the HV backscattering coefficient (figure 2(c)), it increases of about $10 \mathrm{~dB}$ from the initial bare surface scattering up to the bulb growth stage, due to the emergence and development of new leaves, which increase the randomness of the plant structure. Among the three backscattering channels, the cross-poalrized one exhibits the largest dynamic range.

It must be underlined that, for parcel $\mathrm{G}$, trends reported in figure $2(a)-(c)$ are in total agreement with results reported in (Moran et al. 2012) although in that work a different representation is used (plots against DoY).

\subsection{Coherence Between Copolar Channels}

The coherence between the $\mathrm{HH}$ and the $\mathrm{VV}$ complex channels, $\rho_{\mathrm{hhvv}}$, is derived from the elements of $\mathbf{C}(2)$ as (Skriver et al. 1999)

$$
\rho_{\mathrm{hhvv}}=\left|\rho_{\mathrm{hhvv}}\right| e^{j \phi_{\mathrm{hhvv}}}=\frac{\left\langle S_{\mathrm{hh}} S_{\mathrm{vv}}^{*}\right\rangle}{\sqrt{\left\langle\left|S_{\mathrm{hh}}\right|^{2}\right\rangle\left\langle\left|S_{\mathrm{vv}}\right|^{2}\right\rangle}}
$$

where $\phi_{\mathrm{hhvv}}$ is the copolar phase difference, and $|\cdot|$ stands for the modulus.

The evolution of $\left|\rho_{\mathrm{hhvv}}\right|$ (bounded between 0 and 1 ) as a function of phenology is shown in figure $2(e)$.

From stages 0 to 2 , when surface scattering dominates the radar response, we observe, as expected, a high coherence with values between 0.6 and 1 (for parcel D, i.e. beam D36, and parcel G, i.e. beam A39, such values are slightly below 0.6). As the plants foliage develops from 2 to 7 the coherence decreases, as expected, to values between 0 and $\sim 0.4$. However, if we focus on the evolution of beams A23 and D25 for parcels E, F and G, it presents higher values with respect to the evolution of the other beams, and to the evolution of the same beams for the other parcels. Regarding beam D25 around stage 3, the larger $\left|\rho_{\text {hhvv }}\right|$ values obtained over parcel $\mathrm{E}(>0.7)$ and parcel $\mathrm{G}(>0.6)$ are due to irrigation. The irrigation 
schedule, provided only for these two fields, reports that, in that day (May 21, DoY 141), both fields were irrigated; with about 8 (6) $\mathrm{mm}$ of water for parcel $\mathrm{E}$ (parcel G). Moreover, parcel G was also irrigated on June 14 (DoY 165) when beam D25 was collected (between stages 5 and 6). Therefore the larger backscattering from the ground led to an increase of $\left|\rho_{\mathrm{hhvv}}\right|$, that, as expected is more noticeable at steeper incidence angles. With respect to parcel $\mathrm{F}$, which results in a larger coherence value $\left(\left|\rho_{\text {hhvv }}\right|>0.8\right)$, unfortunately no information about irrigation is provided. Concerning beam A23, neither parcel E nor parcel G were irrigated when the images were acquired. Therefore, a possible explanation of the high coherence values exhibited by the beam A23 over parcels E and G can be related to the row orientation of the parcels (see table 2) with respect to the line of sight of the radar. In fact, when dealing with row planted fields, the backscattering at the copolar channels may be significantly affected by the row orientation (Ulaby et al. 1986). As a consequence, the coherence between the copolar channels should be affected as well. This could explain those high coherence values that, otherwise, can not be explained, since, according to ground measurements, they are not due to rain events or irrigation.

From stages 7 to $8\left|\rho_{\text {hhvv }}\right|$ remains mainly below 0.4 , since plants exhibit a fully developed foliage. Regarding the ripening stage, the lower-than-expected coherence value is actually due to the fact that probably ground truth information is not enough accurate in this stage. In fact, if plants were affected by desiccation the main contribution would be the soil one, i.e. a Bragg or tilted-Bragg scattering mechanism that results in higher coherence values. The obtained results show only a slight increase of the copolar coherence (up to $\sim 0.5$ ) in the ripening stage and this is due to the fact that probably plants have just started desiccating.

This reasoning is supported by results obtained in the pre-harvest stage, which practically consists of the advanced ripening stage testimated by the knowledge of the harvest date, where desiccated plants result in larger coherence values (up to 0.8). The copolar coherence for parcel $\mathrm{F}\left(\left|\rho_{\mathrm{hhvv}}\right|\right.$ values between 0.6 and 0.8$)$ is larger than the parcel $\mathrm{E}$ one $\left(\left|\rho_{\mathrm{hhvv}}\right|\right.$ values below 0.5). Although, as underlined in the previous subsection, a rain event occurred during the days in where beams A23 and A39 were collected, such an effect can be related to the harvesting (see table 2). In fact, it is quite probable that the harvesting occurred immediately after September 20 for parcel $\mathrm{F}$ while a little later for parcel E. This explains the high values of $\left|\rho_{\mathrm{hhvv}}\right|$ for parcel $\mathrm{F}$, that results mainly in surface scattering.

\subsection{Eigenvalue/Eigenvector Decomposition}

In this subsection, the behavior of the polarimetric observables obtained from the well known eigenvalue/eigenvector decomposition of the coherency matrix (Cloude and Pottier 1996, 1997) is analyzed. Figure 3 shows the evolution, as a function of phenology, of four parameters derived from such decomposition: entropy $H$, anisotropy $A$, average scattering angle $\bar{\alpha}$, and dominant scattering angle $\alpha_{1}$.

First, we note that those observables exhibit a clear trend that allows to interpret the radar response of the parcels in terms of the scattering mechanisms associated to different stages of plants development. Moreover, according to the behavior of the copolar coherence shown in figure 2(e), the evolution of the entropy, $\bar{\alpha}$ and $\alpha_{1}$ relevant to beams A23 and D25 for parcels E, F and G is characterized by lower values with respect to the other cases, due to irrigation and row orientation.

From the seedling to the $3 / 4$ leaves stage $(0-4) H$ increases from values between 0.2 and 0.6 to values between 0.7 and 0.9 , whereas $A$ assumes lower values (between 
0.2 and 0.5 with a peak around 0.6 ). The lowest $H$ values at the beginning of the growing season indicate the presence of a single scattering mechanism that can be interpreted as surface-type, according to $\bar{\alpha}$ and $\alpha_{1}$. Then, as plants develop up to stage 4, $H$ increases as a consequence of leaves emergence. The larger $H$ values observed at the end of this fourth stage witness that the scattering from an almost random volume is occurring. In fact, four leaves are fully emerged (see table 1) with the older (outer) leaves, i.e. the second and the third, very tilted. Regarding the average and the dominant scattering angle at this stage, values of $\bar{\alpha}$ between $30^{\circ}$ and $50^{\circ}$ are observed, while $\alpha_{1}$ is mainly below $20^{\circ}$, denoting that the dominant scattering mechanism is still the surface one.

From stages 4 to $9 \mathrm{H}$ tends to 0.9 , reaching its maximum at the end of ripening. Concerning $A$, it decreases up to values below 0.3. This trend is due to the high random structure of the canopy, caused by the emergence of further leaves and then by the bending of the leaves in the bulb growth stage. Therefore, the largest depolarization experienced at these stages is clearly switnessed by these polarimetric observables.

Up to the middle of the bulb growth stage, $\bar{\alpha}$ is mostly between $40^{\circ}$ and $50^{\circ}$, while $\alpha_{1}$ increases from a range of $10^{\circ}-20^{\circ}$ to a range of $20^{\circ}-40^{\circ}$. This means that the dominant scattering mechanism corresponds to the scattering from anisotropic surfaces, i.e. a surface-type scattering where the term anisotropic refers to the difference, in terms of magnitude, between $\mathrm{HH}$ and VV. Then, as plants go in through the ripening stage, $\alpha_{1}$ decreases since a pure surface scattering starts to dominate the radar echo again.

Finally, in agreement with the copolar coherence, at the end of the pre-harvest stage we observe a larger decrease of both $H$ and $\bar{\alpha}$ for parcel $\mathrm{F}$ with respect to parcel E. Regarding $\alpha_{1}$, it ranges between $10^{\circ}$ and $20^{\circ}$ for parcel $\mathrm{E}$ and it is below $10^{\circ}$ for parcel $\mathrm{F}$. Hence, due to the time gap between the harvesting of the two parcels, only for parcel $\mathrm{F}$ we observe one scattering mechanism, i.e. surface scattering.

\section{Retrieval of phenological stages}

Phenological stages of agricultural crops can be mapped into different classes whose identification can be considered a classification problem (Lopez-Sanchez et al. 2012, 2014). On this purpose, in this section both single-polarization and polarimetric observables are exploited to classify phenological stages of onion fields. Since the single-polarization analysis showed that the HV channel is the most sensitive to the onion phenology, $\mathrm{HH}$ and VV channels will not furthered.

Three phenological intervals can be easily identified for onion fields. They results from a partitioning of the whole vegetative phase reported in table 1 into three main phases:

(1) Early vegetative phase: from seeding to 2 leaves stage (phenological interval $0-2)$.

(2) Middle vegetative phase: from $2 / 3$ leaves stage to $6 / 7$ leaves stage (phenological interval 2-6).

(3) Advanced vegetative phase: from start of bulb growth to ripening/pre-harvest stages (phenological interval 6-10).

By adopting the methodology described in (Lopez-Sanchez et al. 2012, 2014), these intervals can be retrieved using a very simple classification algorithm. 
It must be noted that, in order to obtain an estimation as correct as possible, none of the polarimetric observables can be used alone.

Therefore, different polarimetric observables need to be jointly used to obtain a satisfactory phenology retrieval. In this study, the HV backscattering coefficient and $\left|\rho_{\text {hhvv }}\right|$ are used. The joint use of these two parameters allows to reduce ambiguities between the three phenological intervals. If $\left|\rho_{\text {hhvv }}\right|$ is used alone, the early and the middle vegetative phases would be easily distinguished since they result in large coherence values. However, we would experience a total ambiguity between the middle and the advanced vegetative phases, due to decrease of this parameter with the foliage development occurring at these intervals (see figure $2(e)$ ). On the other hand, if the sole $\mathrm{HV}$ backscattering coefficient is used, many ambiguities are observed. The joint use of $\left|\rho_{\mathrm{hhvv}}\right|$ and the HV backscattering coefficient allows a correct and reliable phenology retrieval. In fact, $\left|\rho_{\mathrm{hhvv}}\right|$ turns out to be fundamental to separate the first two phenological intervals; while, the HV backscattering coefficient, resulting in large values in the advanced vegetative phase due to the large randomness of the foliage, allows separating the middle and the advanced vegetative phases.

In figure 4(a) the plane defined by all the measured parameters (mean and standard deviation) of this pair of observables is shown, and the early, the middle and the advanced vegetative phase are colored in blue, green and red, respectively. Note that the three intervals are generally not overlapped. The mixing and overlapping values that we observe regard those days in which the values of the polarimetric features do not meet the expected behavior. As a consequence, these points are expected to be misclassified. In some occasions, misclassifications are due to values very close to the transitions between phenological intervals. This is unavoidable since, usually, different zones of a field develop at different rates. In other words, such transitions are not so abrupt in nature.

The three phenological intervals are retrieved by considering the decision plane that is obtained by thresholding the $\left|\rho_{\text {hhvv }}\right|-\mathrm{HV}$ space, as shown in figure $4(b)$. It is important to underline that, since in this study the estimation of phenology is based on physically based observables, the algorithm is designed to be as simple as possible. For such a purpose, thresholds are set manually.

The classification algorithm is applied to all the available images, i.e. to all the beams, and the classification is carried out at pixel level: for each image, a pixel is assigned to one of the three phenological intervals if it falls in the corresponding region identified in the $\left|\rho_{\mathrm{hhvv}}\right|-\mathrm{HV}$ decision plane. Those pixels that fall outside the three regions are not classified. Then, once all the pixels of a given parcel have been classified, the percentage of pixels assigned to each phenological interval is computed and the mode, i.e. the most retrieved value, is used to decide the parcel phenology.

The performance of the estimation is assessed by comparing the retrieved phenological intervals with the available ground truth. This comparison allows defining a confusion matrix by updating the diagonal elements when the phenological intervals of each parcel in every acquisition day are correctly estimated, or the off-diagonal ones when wrong estimations (misclassifications) occur. The calculation of the confusion matrix allows obtaining the Overall Accuracy (OA) and both Producers Accuracy (PA) and Users Accuracy (UA) for each of the three classes The confusion matrix, relevant to all the parcels, is shown in table 7 , where also the OA, the PA, the UA and the Kappa coefficient are listed.

The OA is $86.23 \%$ with Kappa $=0.78$. We note that, while the early and the advanced vegetative phases are characterized by an high PA, the middle vegetative 
phase results in the worst PA, as a consequence of the higher number of misclassifications between this class and the others. Regarding the UA, it exhibits its lowest value in the middle vegetative phase.

It must be noted that other polarimetric observables can be used to estimate the three phenological intervals. For instance, if only $H$ is considered (see figure 3(a)), we note that the lowest values experienced in the early vegetative phase would allow to identify this phase. Regarding the middle and the advanced vegetative phases, it is evident that they cannot be estimated with $H$ alone. Therefore, even in this case a joint use of $H$ and the HV backscatter is needed to obtain a correct and reliable retrieval. The $H-\mathrm{HV}$ space and the corresponding decision plane are not shown to save space. In table 8 the confusion matrix relevant to the estimation undertaken with this pair of observables is shown. The OA is slightly lower (about the $0.4 \%$ ) with respect to the $\left|\rho_{\mathrm{hhvv}}\right|-\mathrm{HV}$ case. This is a consequence of the larger number of misclassifications that is experienced between the middle and the advanced vegetative phases. These misclassifications give rise to a decrease of both the PA in the middle vegetative phase $(\sim 5 \%)$ and the UA in the advanced vegetative phase $(\sim 2.5 \%)$. Regarding the $\mathrm{PA}$ in the early and advanced vegetative phases and the UA in the early and the middle vegetative phases, the pair $H-\mathrm{HV}$ performs better than the pair $\left|\rho_{\text {hhvv }}\right|-H V$.

Finally, in table 9, the confusion matrix relevant to the estimation of these three phases by using only the HV backscattered power is reported for reference purposes. The classification is undertaken by thresholding the HV backscattering evolution shown in figure $2(c)$. We note the worst OA, lower of about the $6 \%$ with respect the ones of the $\left|\rho_{\mathrm{hhvv}}\right|-\mathrm{HV}$ and $H-\mathrm{HV}$ cases. This is practically due to the many misclassifications (20 cases out of 55) that occur between the early and the middle vegetative phases, that significantly lower the PA in the early vegetative phase, and the UA in the middle vegetative phase. In fact, in the first phase, the PA is lower of about the 30 and the $32 \%$ with respect to the cases in which the HV backscattering is used jointly with the copolar coherence and the entropy, respectively. Concerning the UA, it is higher of about the $2,5 \%$ than the $\left|\rho_{\mathrm{hhvv}}\right|-\mathrm{HV} / \mathrm{H}-\mathrm{HV}$ ones. In the middle vegetative phase, although the $\mathrm{HV}$ channel provides the best PA, the UA is lower of about the $16 \%(20 \%)$ than the ones provided by the pair $\left|\rho_{\mathrm{hhvv}}\right|-\mathrm{HV}$ $(H-\mathrm{HV})$. Therefore, HV backscattering, as expected, provides the poorest result in the classification of all the three phenological intervals.

An interesting aspect of this analysis regards the dependence of $\left|\rho_{\mathrm{hhvv}}\right|, H$ and HV backscattering coefficient on the incidence angle. This dependence is explicitly addressed analyzing, for each field, the behavior of these features for all the available beams. Main results are commented as follows. Regarding the HV backscattering coefficient, it exhibits a strong variation with incidence angle in the first two phenological intervals, due to the dominance of the ground response. Such a variation is negligible in the third phenological interval. Concerning $\left|\rho_{\mathrm{hhvv}}\right|$ and $H$, their variation with the incidence angle is less significant in the first and the third phenological intervals with respect to the second one. In the first phenological interval the $\left|\rho_{\mathrm{hhvv}}\right|$ is manly above 0.6 while $H$ is mainly below 0.6 , with the lowest incidence angles exhibiting the largest $\left|\rho_{\mathrm{hhvv}}\right|$ and the lowest $H$ values. In the second phenological interval, as expected, $\left|\rho_{\mathrm{hhvv}}\right|$ decreases as the incidence angles increases, while $H$ increases. Finally, in the third phenological interval the coherence is mainly below 0.4 (except those values evaluated in the pre-harvest stage for parcels $\mathrm{E}$ and $\mathrm{F}$ ) while $H$ is mainly above 0.8 .

In summary, the vegetative phase of onion fields, divided in three main intervals, is estimated with high accuracies by considering not only the conventional measur- 
ables (backscattering coefficients), but meaningful polarimetric observables, such as the coherence between the copolar channels and the entropy.

\section{Conclusions}

In this study, for the first time, polSAR observables derived from C-band SLC quad-pol RADARSAT-2 images are used to estimate phenological stages of onion fields in the first year of growth. Three main phenological intervals are defined and a performance analysis is undertaken using both polarimetric observables and conventional intensity features. Experimental results show that the joint use of polarimetric features and the cross-polarized intensity results in the best retrieval of the three phenological intervals.

It would be interesting to estimate, in a future study, the phenological stages of onion during the second year of growth (reproduction and maturation phases) if both polSAR time series and ground measurements were available in this growing season.

\section{Acknolodegment}

This work was partially supported by the Spanish Ministry of Economy and Competitiveness (MINECO) and EU FEDER, under Project TEC2011-28201-C0202. All RADARSAT-2 images were provided by ESA in the framework of the AgriSAR2009 campaign. RADARSAT-2 Data and Products (c) MacDonald, Dettwiler and Associates Ltd. (2009) - All Rights Reserved. RADARSAT is an official trademark of the Canadian Space Agency. 


\section{References}

Atzbeger, C., Richter, K. (2012), Spatially Constrained Inversion of Radiative Transfer Models for Improved LAI Mapping from Future Sentinel-2 Imagery, Remote Sensing of Environment, 120, 208-218.

Berger, M., Rast, M., Wursteisen, P., Attema, E., Moreno, J., F., Müller, A., Beisl, U., Richter, R., Schaepman, M., Strub, G., Stoll, M., P., Nerry, F., Leroy, M. (2001), The DAISEX Campaigns in Support of a Future Land-Surface-Processes Mission, ESA Bulletin, 105, 101-111.

Brewster, J. L. (2008), Onions and other vegetable Alliums, CABI.

Cloude, S.,R., And Pottier, E. (1996), A Review of Target Decomposition Theorems in Radar Polarimetry, IEEE Transaction on Geoscience and Remote Sensing, 34, 498518.

Cloude, S.,R., And Pottier, E. (1997), An Entropy Based Classification Scheme for Land Applications of Polarimetric SAR, IEEE Transaction on Geoscience and Remote Sensing, 35, 68-78.

De Dernanrdis, C., Vicente-Guijalba, F., Martinez-Marin, T., LopezSanchez, J. M.. (2014), Estimation of Key Dates and Stages in Rice Crops Using Dual-Polarization SAR Time Series and a Particle Filtering Approach, IEEE Journal of Selected Topics in Applied earth Observations and Remote Sensing, DOI: 10.1109/JSTARS.2014.2372898

Gonzáles-Sanpedro, M., C., Le Toan, T., Moreno, J. F. (2005), Multitemporal Analysis of MERIS-FR Data for Crop Monitoring in two Agricultural Areas: Barrax and Tolouse, Proceedings of the 2004 Envisat and ERS Symposium, Salzburg, Austria, SP-572, 6-10 September 2004.

Gonzáles-Sanpedro, M., C., Le Toan, T., Moreno, J. F., Kergoat, L., RuBIO, E. (2008), Seasonal variations of leaf area index of agricultural fields retrieved from Landsat data, Remote Sensing of Environment, 112, 810-824.

Latorre-Carmona, P., Knyazikhin, Y., Alonso, L., Moreno, J.F., Pla, F.,and YAN, Y. (2014), On Hyperspectral Remote Sensing of Leaf Biophysical Constituents: Decoupling Vegetation Structure and Leaf Optics Using CHRISPROBA Data Over Crops in Barrax, IEEE Geoscience and Remote Sensing Letters, 11, 1579-1583.

Lee, R., Baldwin, S., Kenel, F., McCallum, J., And MaCKINIGHT, R. (2013), FLOWERING LOCUS T genes control onion bulb formation and flowering, Nature Communications, 4, 1-9.

Le Toan,, T., Laur, H., Mougin, E., and Lopes, A. (1989), Multitemporal and Dual-Polarization Observations of Agricultural Vegetation Covers by X-Band SAR Images, IEEE Transaction on Geoscience and Remote Sensing, 27, 709-718.

LiU, J. S., Paris, W. V., and McNairn, H. (2013), Multiyear Crop Monitoring Using Polarimetric RADARSAT-2 Data, IEEE Transaction on Geoscience and Remote Sensing, 51, 2227-2240.

Lopez-Martinez, C., Pottier, E., And Cloude, S.,R. (2005), Statistical assessment of eigenvector-based target decomposition theorems in radar polarimetry, IEEE Transaction on Geoscience and Remote Sensing, 43, 2058-2074.

Lopez-Sanchez, J. M., Ballester, J. D., And Hajnsek, I. (2011), First Results of Rice Monitoring Practices in Spain by Means of Time Series of TerraSAR-X Dual-Pol Images, IEEE Journal of Selected Topics in Applied earth Observations and Remote Sensing, 4, 412-422.

Lopez-Sanchez, J. M., Cloude, S.,R., Ballester, J. D. (2012), Rice Phenology Monitoring by Means of SAR Polarimetry at X-Band, IEEE Transaction on Geoscience and Remote Sensing, 50, 2695-2709.

Lopez-Sanchez, J. M., Ballester, J. D.,Vicente-Guijalba, F., And Cloude, S.,R. (2013), Estimating Phenology of Agricultural Crops from Space, Proceedings of ESA Living Planet Symposium,Edinburgh, UK, ESA SP-722, 9-13 
September 2013.

Lopez-Sanchez, J. M., Vicente-Guijalba, F., Ballester, J. D., Cloude, S.,R. (2014), Polarimetric Response of Rice Fields at C-Band: Analysis and Phenology Retrieval, IEEE Transaction on Geoscience and Remote Sensing, 50, 2977-2993.

Mascolo, L., Lopez-Sanchez, J. M.,Vicente-Guijalba, F., Mazzarella, G., Nunziata, F., Migliaccio, M. (2014), On the Sensitivity of Polarimetric SAR Observables to Phenological Stages of Crops in Barrax, Spain: First Results at C-Band, Proceedings of the IEEE GOLD Conference, Berlin, Germany, June 5-6, 2014.

Mattia, F., Le Toan, T., Picard, G.,Posa, F. I.,D'Alessio, A., Notarnicola, C., Gatti, A, M.,Rinaldi, M., Satalino, G., and Pasquariello, G. (2003), Multitemporal C-Band Radar Measurements on Wheat Fields, IEEE Transaction on Geoscience and Remote Sensing, 41, 1551-1560.

MeIER, U. (2001), Growth Stages of Mono-and Dicotyledonous Plants, Biologic Research Center for Agriculture and Forestry, Online: http://www.bba.de/veroeff/bbch/bbcheng.pdf

Moran, M. S., Alonso, L., Moreno, J. F., Mateo, M., P., C., De la Cruz, D., F.,And Montoro , A. (2012), A RADARSAT-2 Quad-Polarized Time Series for Monitoring Crop and Soil Conditions in Barrax, Spain, IEEE Transaction on Geoscience and Remote Sensing, 50, 1057-1070.

Opara, L., U. (2003), ONION: Post-Harvest Operations, FAO, Online: http://www.fao.org/fileadmin/user_upload/inpho/docs/Post_Harvest_Compendium_-Onion.pdf.

Skriver, H., Svendsen, M., T., And Thomesen, A., G. (1999), Multitemporal C- and L- band polarimetric signatures of crops, IEEE Transaction on Geoscience and Remote Sensing, 37, 2413-2429.

Sobrino, J., A., Franch, B., Oltra-Carrió, R., Vermote, E.F., And FEDELE, E. (2013), Evaluation of MODIS Albedo product over a heterogeneous agricultural area, International Journal of Remote Sensing, 34, 5530-5540.

Ulaby, F., Moore, R., Fung, A., K. (1986), Microwave Remote Sensing: Active and Passive. vol. 3. Norwood, MA: Artech House.

Vicente-Guijalba, F., Martinez-Marin, T., And LopezSAnchez, J. M.. (2014), Crop Phenology Estimation Using a Multitemporal Model and a Kalman Filtering Strategy, IEEE Geoscience and Remote Sensing Letters, 11, 1081-1085.

Vicente-GuiJalba, F., Martinez-Marin, T., And LopezSAnchez, J. M. (2014), Dynamical Approach for Real-Time Monitoring of Agricultural Crops, IEEE Transaction on Geoscience and Remote Sensing, DOI: 10.1109/TGRS.2014.2372897

Zadoks, J., C., Chang, T., T., And Konzak, C., F. (1974), A decimal code for the growth stages of cereals, Weed Research, 14, 415-421. 
Table 1. Phenological stages of onion parcels provided by the main set of ground measurements. The start and the end date of each stage are different for all the parcels.

\begin{tabular}{|c|l|}
\hline Time window & \multicolumn{1}{|c|}{ Phenological stage } \\
\hline Start date $_{S T A G E_{1}}-$ End date $_{S T A G E_{1}}$ & $S T A G E_{1}:$ Seedling \\
\hline Start date $_{S T A G E_{2}}-$ End date $_{S T A G E_{2}}$ & $S T A G E_{2}: 2$ leaves \\
\hline Start date $_{S T A G E_{3}}-$ End date $_{S T A G E_{3}}$ & $S T A G E_{3}: 2-3$ leaves \\
\hline Start date $_{S T A G E_{4}}-$ End date \\
$S T A G E_{4}$ & $S T A G E_{4}: 3-4$ leaves \\
\hline Start date $_{S T A G E_{5}}-$ End date $_{S T A G E_{5}}$ & $S T A G E_{5}: 5-6$ leaves \\
\hline Start date $_{S T A G E_{6}}-$ End date $_{S T A G E_{6}}$ & $S T A G E_{6}: 6-7$ leaves \\
\hline Start date $_{S T A G E_{7}}-$ End date $_{S T A G E_{7}}$ & $S T A G E_{7}:$ Start of bulb growth \\
\hline Start date $_{S T A G E_{8}}-$ End date $_{S T A G E_{8}}$ & $S T A G E_{8}:$ Bulb growth \\
\hline Start date $_{S T A G E_{9}}-$ End date $_{S T A G E_{9}}$ & $S T A G E_{9}:$ Ripening \\
\hline
\end{tabular}

Table 2. Additional information regarding the parcels analyzed. Horizontal lines $(-)$ denote that the information is not available.

\begin{tabular}{|c|c|c|c|c|c|}
\hline Parcel & Start date $_{\text {Seedling }}(\mathrm{DoY})$ & End date $_{\text {Ripening }}(\mathrm{DoY})$ & $\begin{array}{l}\text { Further information about phenology } \\
\text { (second set of ground measurements) }\end{array}$ & Harvest date (DoY) & Row orientation \\
\hline A & March 30 (89) & August 19 (231) & $\begin{array}{l}\text { September 10 (DoY 253): the parcel is } \\
\text { in the ripening stage }\end{array}$ & - \\
\hline B & March 15 (74) & August 22 (234) & - & - \\
\hline C & March 15 (74) & August 19(231) & - & - \\
\hline D & April 19 (109) & August 19(231) & - & North-South \\
\hline E & March 30 (89) & August 23 (235) & - & North-South \\
\hline F & March 30 (89) & August 23(235) & Since September 20 (263) & NorthEast-SouthWest \\
\hline G & March 30 (89) & August 23 (235) & - & - \\
\hline
\end{tabular}

Table 3. Characteristics of the RADARSAT-2 images.

\begin{tabular}{|c|c|c|c|c|c|c|c|}
\hline & \multicolumn{7}{|c|}{ Fine Quad-Polarization mode images } \\
\hline Beam & FQ4 & FQ6 & FQ9 & FQ11 & FQ14 & FQ16 & FQ20 \\
\hline Orbit & Ascending & Descending & Ascending & Descending & Ascending & Descending & Ascending \\
\hline Average AOI (degrees) & 23 & 25 & 28 & 31 & 34 & 36 & 39 \\
\hline Acquisition time & $\sim 6 \mathrm{pm}$ & $\sim 6: 20 \mathrm{am}$ & $\sim 6 \mathrm{pm}$ & $\sim 6: 20 \mathrm{am}$ & $\sim 6 \mathrm{pm}$ & $\sim 6: 10 \mathrm{am}$ & $\sim 6 \mathrm{pm}$ \\
\hline Radar center frequency & \multicolumn{7}{|c|}{$5.405 \mathrm{GHz}$} \\
\hline Slant-range pixel spacing & \multicolumn{7}{|c|}{$4.73 \mathrm{~m}$} \\
\hline Azimuth pixel spacing & $4.92 \mathrm{~m}$ & $4.69 \mathrm{~m}$ & $4.81 \mathrm{~m}$ & $5.58 \mathrm{~m}$ & $4.76 \mathrm{~m}$ & $5.15 \mathrm{~m}$ & $4.79 \mathrm{~m}$ \\
\hline
\end{tabular}

Table 4. RADARSAT-2 images used for each parcel.

\begin{tabular}{|c|c|c|}
\hline Parcel & Beams that cover the parcel & Number of images used \\
\hline $\mathrm{A}$ & All except FQ20 & 33: from April 3 to August 31 \\
\hline $\bar{B}$ & All except FQ16 and FQ20 & 30: from April 3 to August 21 \\
\hline $\bar{C}$ & All except FQ16 and FQ20 & 27: from April 3 to August 14 \\
\hline$\overline{\mathrm{D}}$ & All except FQ20 & 29: from April 23 to August 15 \\
\hline $\mathrm{E}$ & All & 43: from April 3 to September 18 \\
\hline $\mathrm{F}$ & All & 45: from April 3 to September 18 \\
\hline G & All & 40: from April 9 to August 21 \\
\hline
\end{tabular}

Table 5. Scheme used to build the numerical scale associated to the phenological evolution of the parcels.

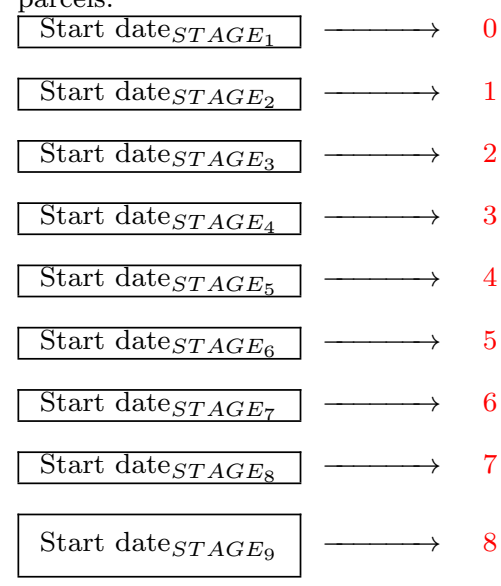

\begin{tabular}{|c|c|}
\hline End date $_{S T A G E_{1}}$ & \\
\hline End date $_{S T A G E_{2}}$ & \\
\hline End date $_{S T A G E_{3}}$ & \\
\hline End date $_{S T A G E_{4}}$ & \\
\hline End date $_{S T A G E_{5}}$ & \\
\hline End date $S T A G E_{6}$ & \\
\hline End date $S T A G E_{7}$ & \\
\hline End date $_{S T A G E_{8}}$ & \\
\hline $\begin{array}{l}\text { End date }_{S T A G E_{9}} / \text { Ad- } \\
\text { ditional information }\end{array}$ & \\
\hline Harvest date & \\
\hline
\end{tabular}


Table 6. Phenology of the onion parcels described in terms of the numerical scale defined in table 5 .

\begin{tabular}{llc}
\hline Stage & Phenology & Numerical scale \\
\hline$S T A G E_{1}$ & Seedling & $0-1$ \\
$S T A G E_{2}$ & 2 leaves & $1-2$ \\
$S T A G E_{3}$ & 2-3 leaves & $2-3$ \\
$S T A G E_{4}$ & 3-4 leaves & $3-4$ \\
$S T A G E_{5}$ & 5-6 leaves & $4-5$ \\
$S T A G E_{6}$ & 6-7 leaves & $5-6$ \\
$S T A G E_{7}$ & Start of bulb growth & $6-7$ \\
$S T A G E_{8}$ & Bulb growth & $7-8$ \\
$S T A G E_{9}$ & Ripening & $8-9$ \\
$S T A G E_{10}$ & Pre-harvest & $9-10$ \\
\hline
\end{tabular}

Table 7. Confusion matrix relevant to the phenology retrieval obtained by the pair $\left|\rho_{\mathrm{hhvv}}\right|-\mathrm{HV}$.

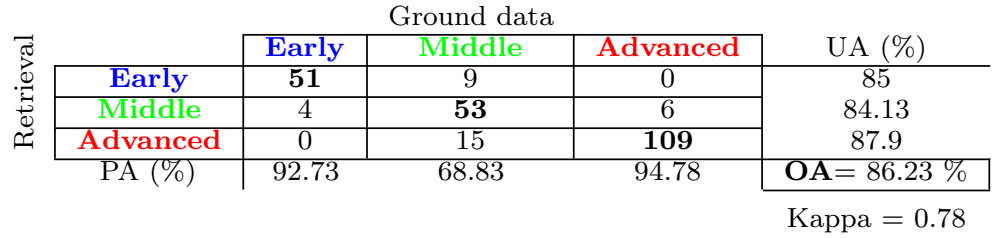

Table 8. Confusion matrix relevant to the phenology retrieval obtained by the pair $H-\mathrm{HV}$.

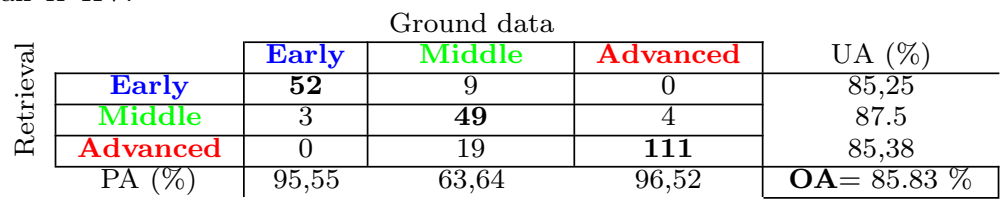

Table 9. Confusion matrix relevant to the phenology retrieval obtained by only the HV backscattering.

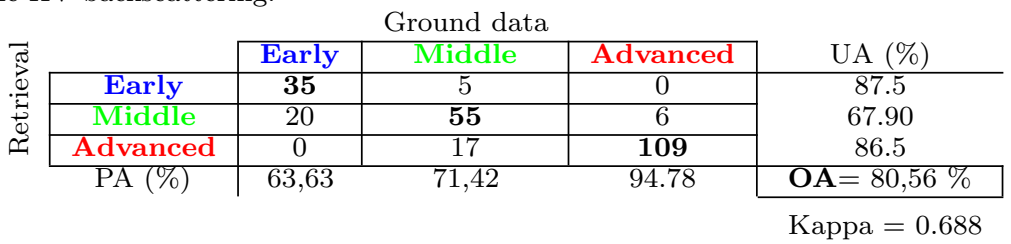




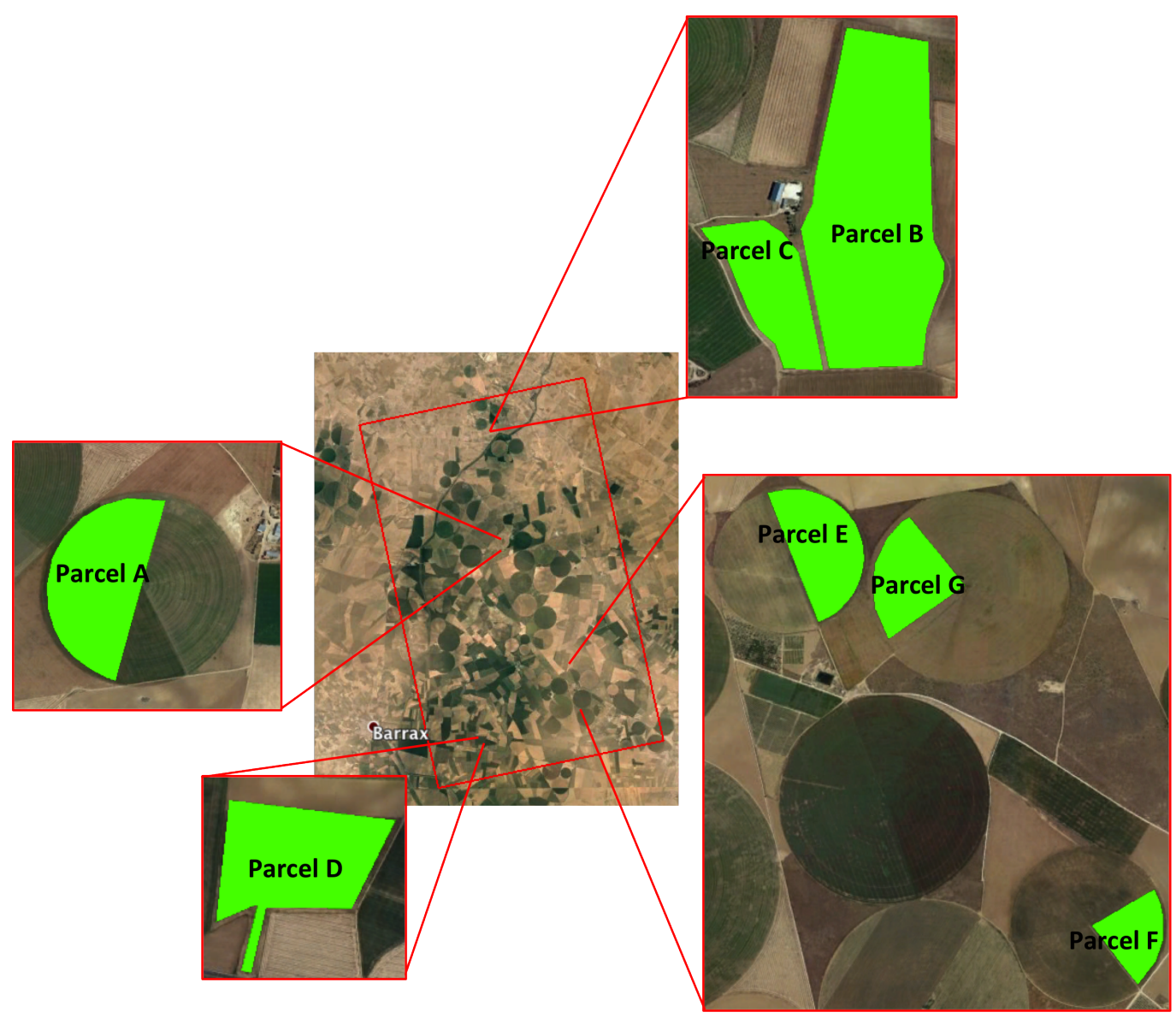

Figure 1. Onion parcels analyzed in this study. Seven fields, highlighted in green, are analyzed. Photos courtesy of Google Earth. 


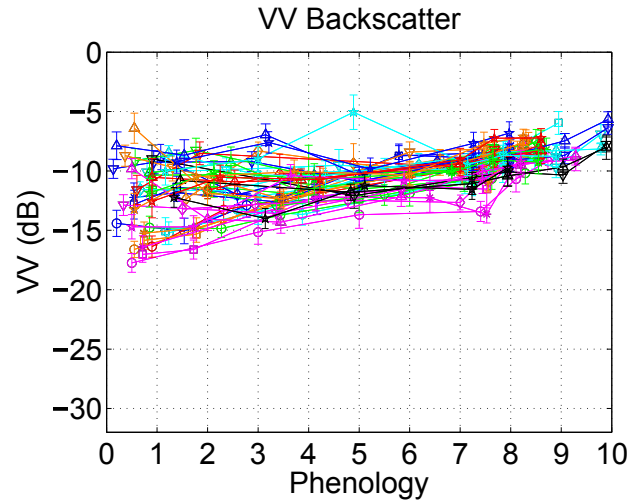

(b)

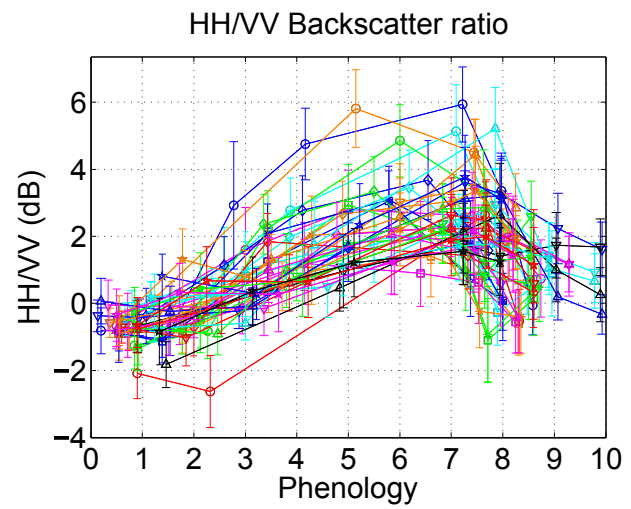

(d)

Figure 2. Behavior, as a function of phenology, of the polarimetric observables derived from the elements of the covariance matrix for the analyzed parcels: (a) HH backscattering coefficient, $(b) \mathrm{VV}$ backscattering

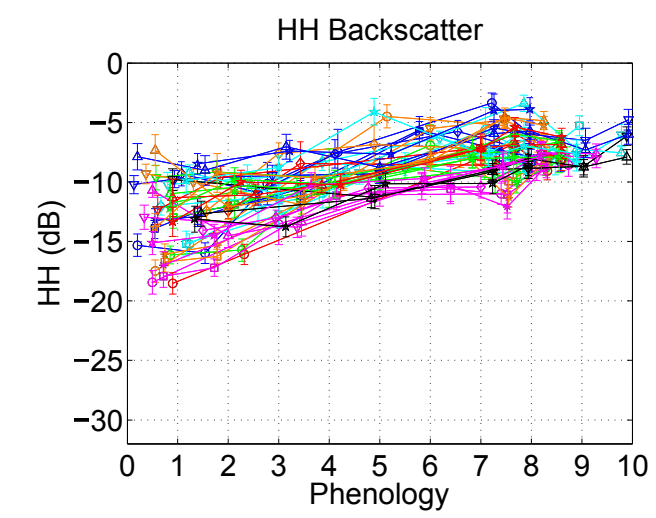

(a)

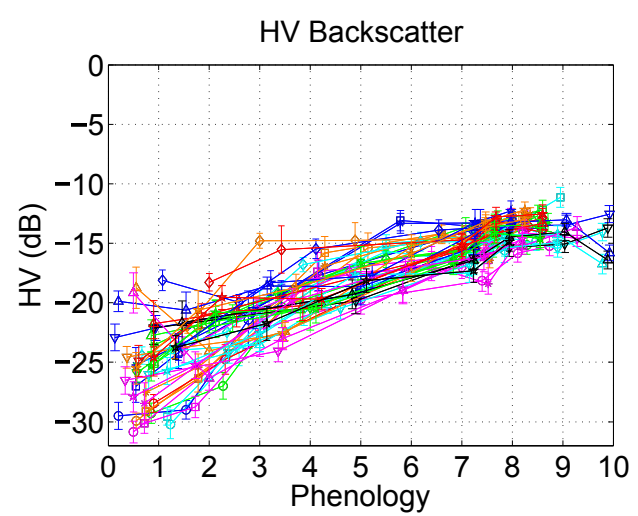

(c)

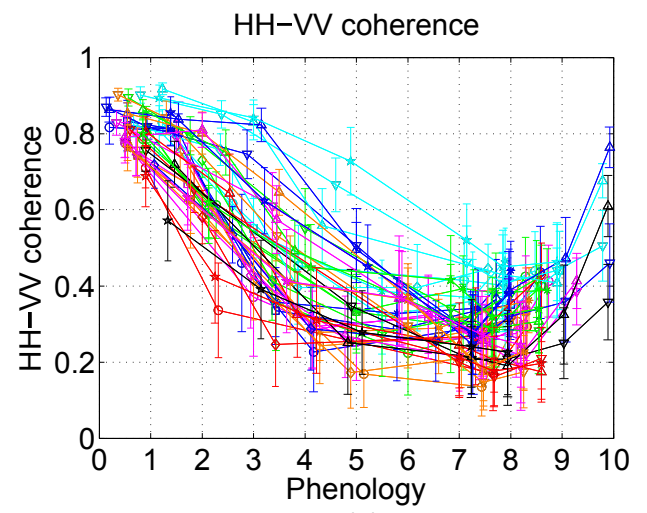

(e)

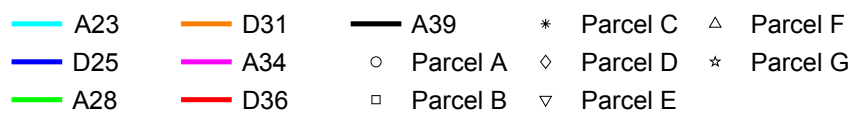

coefficient, (c) HV backscattering coefficient, $(d)$ copolar ratio and $(e)\left|\rho_{\text {hhvv }}\right|$. 


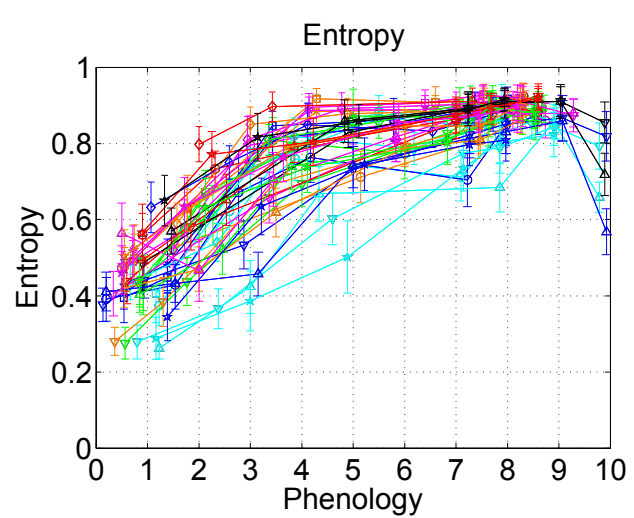

(a)

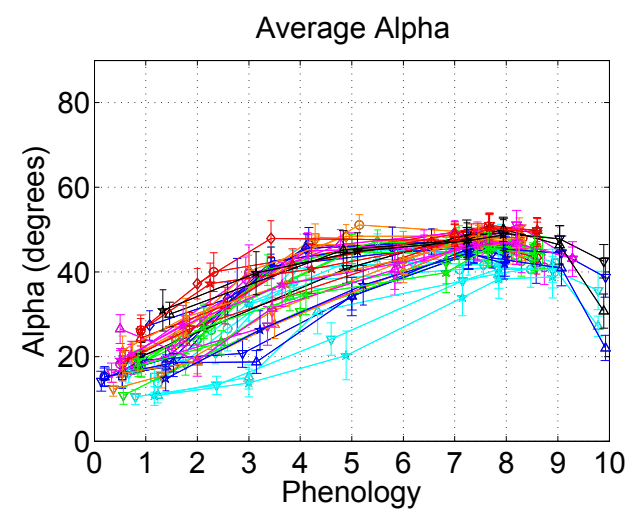

$(c)$

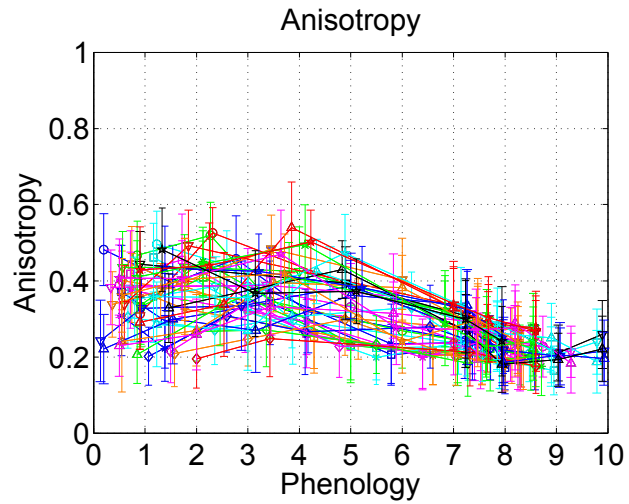

(b)

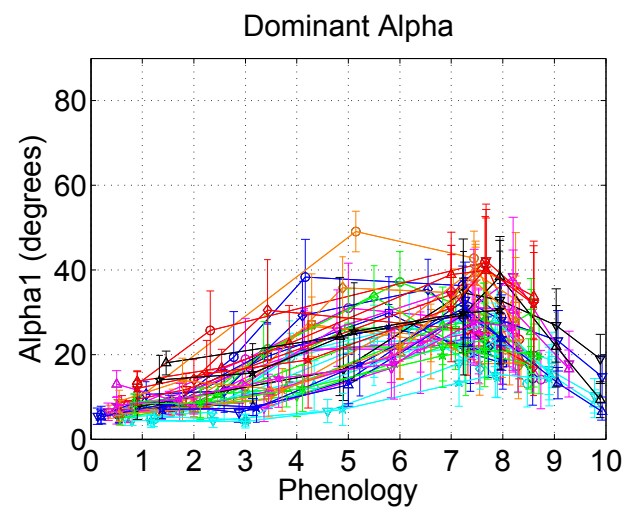

(d)

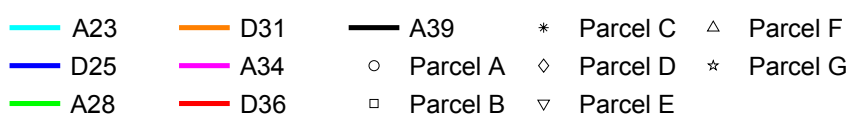

Figure 3. Behavior, as a function of phenology, of the polarimetric observables derived from the eigenvalue/eigenvector decomposition of the coherency matrix for the analyzed parcels: $(a) H,(b) A,(c) \bar{\alpha}$ and (d) $\alpha_{1}$.

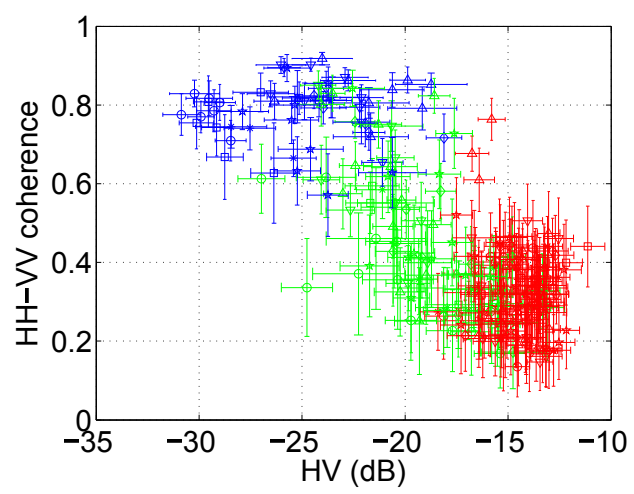

(a)

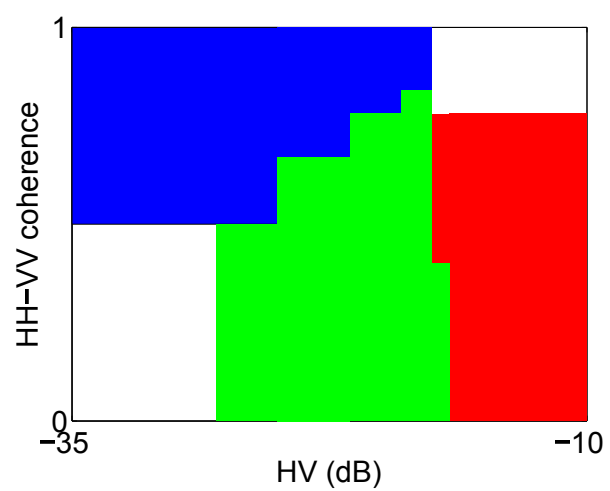

(b)

Figure 4. Scheme used to perform the classification of the three phenological intervals: $(a)\left|\rho_{\mathrm{hhvv}}\right|-\mathrm{HV}$ space and $(b)$ corresponding decision plane. Color coding: Blue: early vegetative; Green: middle vegetative; Red: advanced vegetative. 